\title{
Mixed Lactobacillus plantarum Strains Inhibit Staphylococcus aureus Induced Inflammation and Ameliorate Intestinal Microflora in Mice
}

\author{
Dayong Ren, ${ }^{1}$ Shengjie Gong, ${ }^{1}$ Jingyan Shu, ${ }^{2}$ Jianwei Zhu, ${ }^{1}$ Fengjun Rong, \\ Zhenye Zhang, ${ }^{1}$ Di Wang, ${ }^{1}$ Liangfeng Gao, ${ }^{1}$ Tianming Qu, ${ }^{1}$ Hongyan Liu, ${ }^{1}$ and Ping Chen ${ }^{1}$ \\ ${ }^{1}$ College of Food Science and Engineering, Jilin Agricultural University, Changchun 130118, China \\ ${ }^{2}$ Veterinary Science Department, College of Veterinary Medicine, Jilin University, Changchun 130062, China
}

Correspondence should be addressed to Ping Chen; cchenpin@163.com

Received 1 April 2017; Revised 9 June 2017; Accepted 14 June 2017; Published 27 July 2017

Academic Editor: Silvia Gregori

Copyright (C) 2017 Dayong Ren et al. This is an open access article distributed under the Creative Commons Attribution License, which permits unrestricted use, distribution, and reproduction in any medium, provided the original work is properly cited.

\begin{abstract}
Objective. Staphylococcus aureus is an important pathogen that causes intestinal infection. We examined the immunomodulatory function of single and mixed Lactobacillus plantarum strains, as well as their impacts on the structure of the microbiome in mice infected with Staphylococcus aureus. The experiment was divided into three groups: protection, treatment, and control. Serum IFN$\gamma$ and IL-4 levels, as well as intestinal sIgA levels, were measured during and 1 week after infection with Staphylococcus aureus with and without Lactobacillus plantarum treatment. We used 16s rRNA tagged sequencing to analyze microbiome composition. IFN$\gamma / \mathrm{IL}-4$ ratio decreased significantly from infection to convalescence, especially in the mixed Lactobacillus plantarum group. In the mixed Lactobacillus plantarum group the secretion of sIgA in the intestine of mice $(9.4-9.7 \mathrm{ug} / \mathrm{mL})$ was significantly higher than in the single lactic acid bacteria group. The dominant phyla in mice are Firmicutes, Bacteroidetes, and Proteobacteria. Treatment with mixed lactic acid bacteria increased the anti-inflammatory factor and the secretion of sIgA in the intestine of mice infected with Staphylococcus aureus and inhibited inflammation.
\end{abstract}

\section{Introduction}

Staphylococcus aureus is a major cause of human infection worldwide [1], is a common pathogen in nosocomial infections, and is a zoonotic pathogen [2]. The host produces a large quantity of proinflammatory cytokines and immune regulatory factors in response to pathogen invasion, which can cause multiple organ dysfunction syndrome [3] or even death. S. aureus can express a variety of exotoxins that attack the host immune system [4]. The ratio of Th1 and Th2 cells is important for normal immune response [5]; meanwhile, in an adaptive immune response IFN- $\gamma$ is a subset of $\mathrm{CD}^{+} \mathrm{T}$ helper 1 cells (Thl) that plays an important role in severe inflammation [6] by promoting inflammatory response. S. aureus infection can induce the release of a variety of inflammatory factors, such as IFN- $\gamma$ [7]. S. aureus can also cause intestinal infection. SIgA, which is produced by the intestinal mucosa, can inhibit the invasion of bacteria, can help maintain balance in the body, and has an important protective effect on the intestinal mucosa [8]. S. aureus secretes IgA hydrolase, which helps to inhibit bacterial adhesion [9].

Many lactic acid bacteria (LAB) are probiotics that regulate the body's immune system and help to prevent infection [10]. Studies have shown that some probiotics promote local immunity and IFN- $\gamma$ production while inhibiting IL4 secretion associated with allergies [11]. Lactic acid bacteria induce sIgA secretion, inhibit the invasion of pathogenic bacteria, and protect the host intestinal tract [12]. Some probiotics, such as Lactobacillus plantarum, are involved in mediating immune function [13] and can enhance the immunological function of immunosuppressed mice infected with $S$. aureus. Pro- and anti-inflammatory cytokines and other stress-related signaling molecules play an important role in maintaining balance of immune and inflammatory responses [14]. LAB induce anti-inflammatory factors, such as IL-4 (Th2 cells), while decreasing the levels of enteric 
or serum regulatory factors in the late stages of infection. This results in a lower number of Salmonella in the feces [12]. LAB can inhibit the growth of Escherichia coli, Proteus vulgaris, Enterococcus faecalis, Staphylococcus aureus, and other pathogens under suitable $\mathrm{pH}$ conditions; this may be due to lactate production $[14,15]$.

There have been few studies regarding the ability of LAB to ameliorate $S$. aureus infections. Thus, we screened Lactobacillus plantarum strains in vitro for antibacterial effects abilities (data not shown). L. plantarum was mixed with other LAB to study the effects on immune-related inflammatory factors, sIgA levels, and microbiome composition in mice infected with $S$. aureus. We also verified their abilities to improve immune function and examined whether changes in the immune system are associated with microbiome composition.

\section{Materials and Methods}

2.1. Strains. LAB were extracted from fermented foods. The strains, Lactobacillus plantarum T4 and T8, were mixed $1: 1$ to form mixed lactic acid bacteria. The strains were cultured in MRS medium at $37^{\circ} \mathrm{C}$ for $24 \mathrm{~h}$, centrifuged at $7500 \mathrm{rpm}$ for $2 \mathrm{~min}$, and diluted with $0.9 \%$ sterile saline to $10^{9} \mathrm{CFU} / \mathrm{mL}$. $S$. aureus was obtained from the Institute of Food Science and Engineering Toxicology Laboratory at Jilin Agricultural University and was cultured in $\mathrm{LB}$ medium at $37^{\circ} \mathrm{C}$ for $24 \mathrm{~h}$ and subsequently diluted to $10^{6} \mathrm{CFU} / \mathrm{mL}$. Cultures were stored at $4^{\circ} \mathrm{C}$ until use. At the end of the test, the bacteria were heat sterilized.

2.2. Animals and Feeding. Five-week-old sterile female Kunming mice, weighing $18-20 \mathrm{~g}$, were purchased from the Changchun Institute of Biological Products Co., Ltd. Adequate water was provided, and the mice have free access to chow. Mice were housed in a quiet and ventilated environment; the temperature was $20 \pm 1^{\circ} \mathrm{C}$, the humidity was $50 \% \pm$ $10 \%$, and there was natural light. After 1 week of adaptive feeding, the mice were randomly assigned to cages. There were six mice in each experimental group.

2.3. Experimental Groups. The experiment was divided into the protection, treatment, and control groups. The protection group was, respectively, fed with L. plantarum T4, L. plantarum T8, and mixed LAB for 1 week, followed by 1 week of Staphylococcus aureus. The mice in the treatment group were infected with $S$. aureus for 1 week and then intervened with single and mixed lactic acid bacteria. The negative control group was given $0.9 \%$ sterile saline instead of bacteria. The positive control group was given $S$. aureus for 1 week.

2.4. Pathogen Infection in Mice. S. aureus was delivered at a dose of $10 \mathrm{~mL} / \mathrm{kg}$. Mice were monitored daily. Initial infection was accompanied by lethargy, a reduction in food and water consumption, hair towering, and excretory adhesions; additionally, some sick mice were curled up in a corner. Continued administration led to subcutaneous pustules, part of the epidermis falling off, tail temperature reduced, mice crowding together and not moving, and perianal swelling.
The internal organs of the diseased mice were black, indicating a serious illness in the mice. The mice also had a distinct stench. The control mice did not show any abnormal symptoms.

2.5. Determination of Mouse Organ Index. The mice were weighed, and the liver and spleen were weighed immediately after euthanasia. Organ index was calculated as [12]: Liver or spleen index $=$ [weight of liver or spleen $(\mathrm{g}) /$ body weight $(\mathrm{g})]$ $\times 1000$.

2.6. Cytokine IFN- $\gamma, I L-4$, and Intestinal $\operatorname{sg} A$ Content. Anesthesia was performed by intraperitoneal injection (200 mg/kg/mouse) of a $20 \%$ amobarbital and sterile saline solution (1:3 v:v ratio). Eyeballs were removed for blood collection and centrifuged at $3500 \mathrm{rpm}$ for $10 \mathrm{~min}$, and the supernatant was collected. One centimeter of the colon was rinsed with sterile saline solution. The intestinal contents were collected and stored at $-80^{\circ} \mathrm{C}$ until use. Serum IFN- $\gamma$ and IL4 levels were determined by ELISA using anti-mouse IFN- $\gamma$, IL-4, and biotinylated secondary antibodies according to the manufacturer's instructions. Intestinal sIgA levels were also measured by ELISA. Detection limits for IFN- $\gamma$, IL- 4 , and sIgA ELISA assay, respectively, are $50 \mathrm{pg} / \mathrm{mL}-1500 \mathrm{pg} / \mathrm{mL}$, $0.5 \mathrm{pg} / \mathrm{mL}-100 \mathrm{pg} / \mathrm{mL}$, and $200 \mathrm{ng} / \mathrm{mL}-60000 \mathrm{ng} / \mathrm{mL}$. IFN- $\gamma$, IL-4, and sIgA ELISA kits were purchased from Shanghai Lang Dun Technology Co.

2.7. Analysis of Microbial Structure in Excreta of Mice. Microbial DNA was extracted from mouse feces using an Omega kit according to manufacturer's instructions. Extracted DNA was stored at $-20^{\circ} \mathrm{C}$ until use. Samples were sent to the Shanghai Meji Biomedical Technology Co., Ltd., for sequencing using an Illumina sequencing platform. PCR amplification was carried out using 16s rRNA V3-V4 universal primers with barcodes. The primers were $338 \mathrm{~F}\left(5^{\prime}\right.$ ACTCCTACGGGAGGCAGCAG-3 ${ }^{\prime}$ ) and 806R (5'-GGACTACHVGGGTWTCTAAT-3' ${ }^{\prime}$ ).

2.8. Data Analysis. All results are expressed as mean \pm standard error of the mean (SEM). Statistical analysis was performed using Graphpad Prism 6.0 software. Cytokines were analyzed by two-way ANOVA, and Dunnett's method was used for group comparison. $P$ values less than 0.05 were considered significant.

\section{Results}

3.1. Changes in Body Weight in Mice. The weight of all mice receiving $L$. plantarum increased in the third week (Figure 1(a)), especially the mixed lactic acid bacteria group, which had the most significant increase in body weight (32.0 g) and was closest to the control group (36.5 g). Moreover, the athletic abilities, fecal shape, and fur color of the mice returned to normal. LAB did not significantly alter the body weight of the $S$. aureus infected mice (Figure 1(b)). This indicates that the protection group, especially those treated with mixed $L A B$, recovered from infection. 
TABLE 1: Organ weights of mice in different groups in the first, second, and third weeks of the experiment. ${ }^{*} P<0.05$ versus Con; ${ }^{* *} P<0.01$ versus Con.

\begin{tabular}{lccccrc}
\hline & \multicolumn{2}{c}{ Week 1 } & \multicolumn{2}{c}{ Week 2 } & \multicolumn{2}{c}{ Week 3 } \\
& Liver & Spleen & Liver & Spleen & Liver & Spleen \\
\hline Con & $48.15 \pm 0.17$ & $4.61 \pm 0.99$ & $48.61 \pm 1.99$ & $4.55 \pm 1.12$ & $47.87 \pm 5.65$ & $4.62 \pm 0.41$ \\
\hline P & & & & & & \\
T4 & $47.26 \pm 0.18$ & $4.54 \pm 1.54$ & $48.34 \pm 7.54$ & $4.58 \pm 1.15$ & $49.09 \pm 5.32$ & $5.45 \pm 0.88$ \\
T8 & $47.05 \pm 1.34$ & $4.61 \pm 2.08$ & $46.61 \pm 5.28$ & $4.35 \pm 1.40$ & $48.82 \pm 3.21$ & $4.22 \pm 0.81$ \\
Mix & $48.57 \pm 0.42$ & $4.50 \pm 1.40$ & $49.50 \pm 9.40$ & $3.87 \pm 0.23$ & $49.53 \pm 8.90$ & $4.22 \pm 0.57$ \\
\hline T & & & & & & \\
T4 & $56.73 \pm 0.79^{*}$ & $4.62 \pm 1.23$ & $49.62 \pm 4.23$ & $4.31 \pm 2.02$ & $54.00 \pm 5.53$ & $4.59 \pm 1.77$ \\
T8 & $58.87 \pm 0.38^{*}$ & $4.83 \pm 2.10$ & $54.97 \pm 9.10$ & $4.77 \pm 2.02$ & $53.93 \pm 6.57$ & $4.58 \pm 0.26$ \\
Mix & $58.43 \pm 1.15^{* *}$ & $4.72 \pm 0.80$ & $52.06 \pm 2.80$ & $4.62 \pm 0.78$ & $43.33 \pm 9.01$ & $4.35 \pm 0.88$ \\
\hline Mod & $58.18 \pm 1.14^{* *}$ & $4.67 \pm 1.78$ & $56.18 \pm 5.37^{*}$ & $4.76 \pm 1.83$ & $51.77 \pm 4.70$ & $5.06 \pm 0.97$ \\
\hline
\end{tabular}

The percentage of each animal organ relative to body weight presented as mean \pm SD. Significantly different $(P<0.05)$ values in each column are noted by a $*$. Protection group $=\mathrm{P}$, treatment group $=\mathrm{T}$, and control group $=\mathrm{Con}$. Mix $=$ mixed LAB. Mod $=$ model group. Data is presented as mean \pm SD $(n=6)$. In the first week, one mouse died in the treatment T8 group; data is presented as mean $\pm \mathrm{SD}(n=5)$.

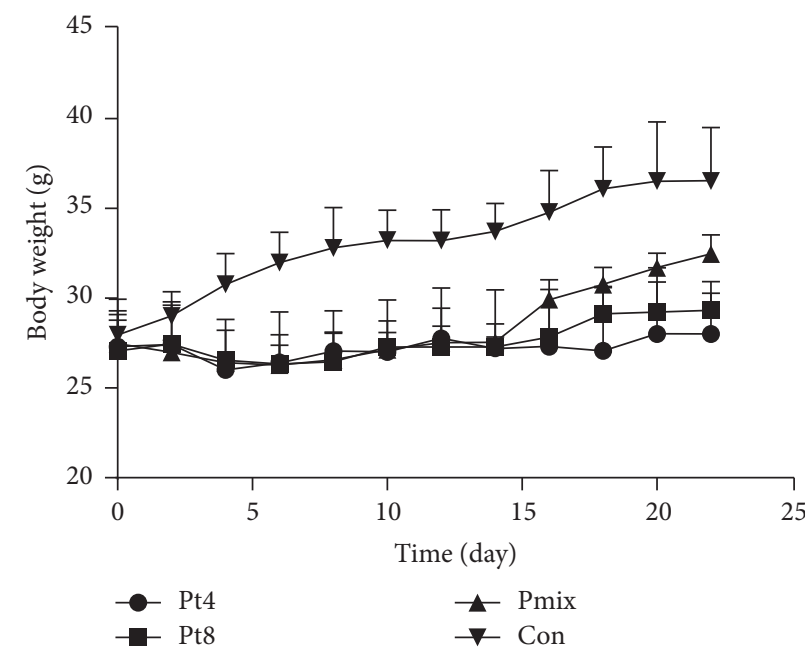

(a)

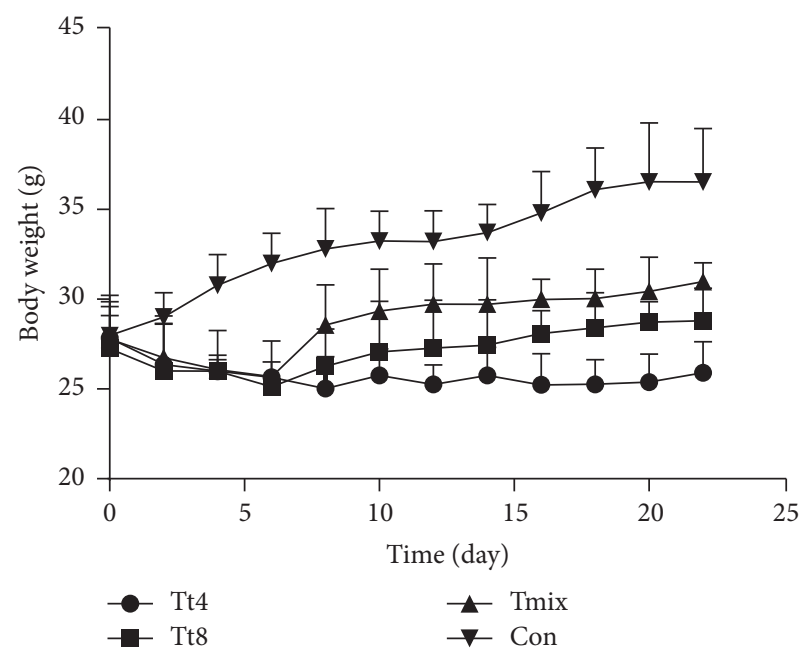

(b)

FIGURE 1: Body weight over time: (a) the protection group (P) containing mice fed Lactobacillus spp. (T4, T8, or Mix) for 1 week, followed by a week of $S$. aureus and then normal feeding for a week, and (b) the treatment group (T) containing mice fed with $S$. aureus for 1 week, followed by a week of Lactobacillus spp. (T4, T8, or Mix) for 1 week and then normal feeding for a week. The control group (Con) did not receive any processing. Mix: mixed LAB. Data were calculated as mean $\pm \mathrm{SD}(n=6)$.

3.2. Organ Index. The liver index of the mice in the protection group was not significantly different from the control group (48.1, 4.6; Table 1). The mice in the treatment group had liver indices $(57.5,4.7)$ that were significantly higher than those of the control group $(19 \%, P<0.05)$. Liver index decreased after 1 week of intervention but was still higher than in the control group $(8 \%, P<0.05)$. Notably, there is one mouse that died during the $S$. aureus infection in the treatment group.

3.3. Serum IFN- $\gamma$ and IL-4 Levels. Compared with the control group, IFN- $\gamma$ was significantly increased $(270-320 \mathrm{pg} / \mathrm{mL}$, $P<0.001)$ during infection in both the protection and the treatment groups Figure 2(a). IL-4 did not change significantly (Figure 2(b)). During recovery, IFN- $\gamma$ significantly decreased $(110-255 \mathrm{pg} / \mathrm{mL}, P<0.001)$ and IL-4 significantly increased $(9-12 \mathrm{pg} / \mathrm{mL}, P<0.001)$. IFN- $\gamma / \mathrm{IL}-4$ ratio decreased significantly from infection to convalescence, especially in the mixed LAB group $(P<0.001)$ Figure $2(\mathrm{c})$.

3.4. Levels of sIgA in Intestine Contents. Levels of sIgA in the intestine contents are shown in Figure 3. Compared with the model group, lactic acid bacteria could increase the secretion of sIgA in the intestine of mice infected with $S$. aureus, especially in the mixed lactic acid bacteria group (9400-9700 $\mathrm{ng} / \mathrm{ml})$, which was significantly higher than that of the single lactic acid bacteria group $(3600-6700 \mathrm{ng} / \mathrm{ml}$, 


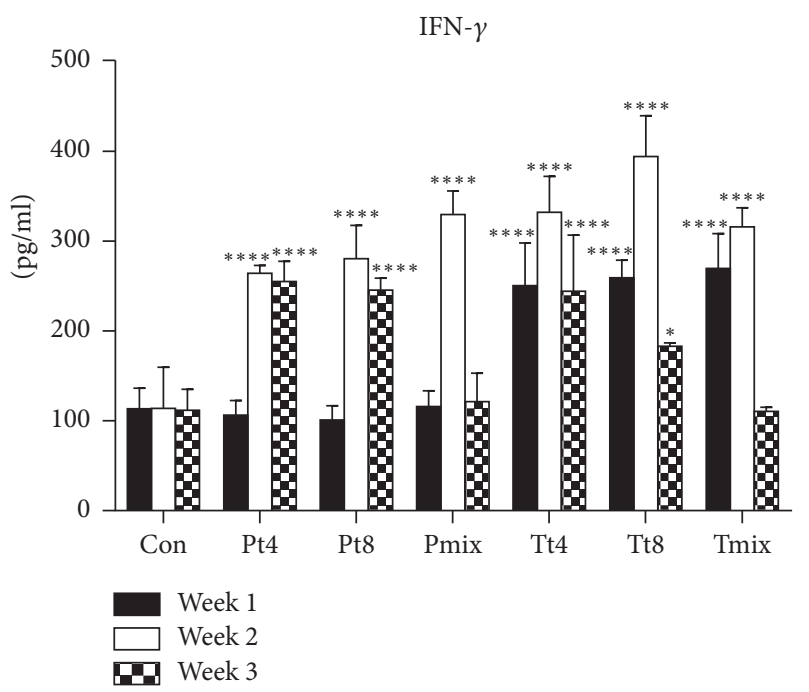

(a)

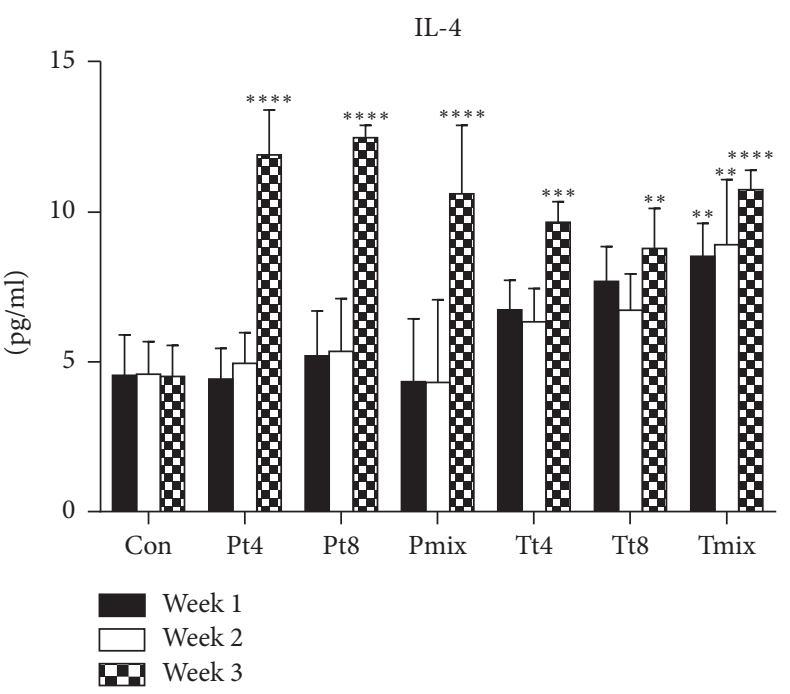

(b)

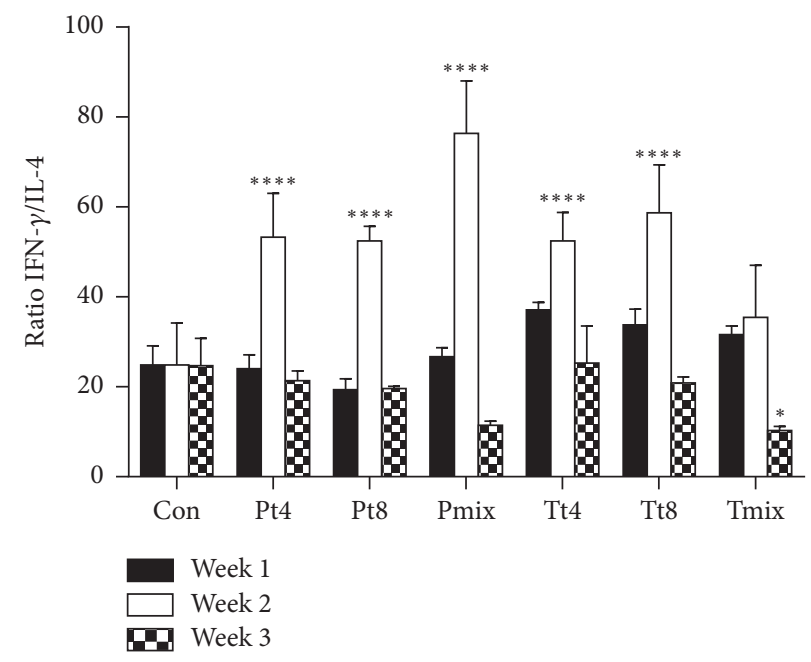

(c)

FIGURE 2: Concentrations and ratios of cytokines in the serum of mice, including serum IFN- $\gamma$ levels (a), serum IL-4 levels (b), and IFN- $\gamma /$ IL4 ratio (c). ${ }^{*} P<0.05,{ }^{* *} P<0.01,{ }^{* * *} P<0.001$, and ${ }^{* * * *} P<0.0001$ versus control. Con: control group, P: protection group, T: treatment group; T4, T8: Lactobacillus plantarum strains; Mix: mixed LAB. Data are presented as mean $\pm \operatorname{SD}(n=6)$.

$P<0.05)$, both in the protection group and in the treatment group.

3.5. Microbiome Structure. The predominant phyla in mice were Firmicutes, Bacteroidetes, and Proteobacteria, and six different phyla were identified (Figure 4). In the control group, the composition was Firmicutes (44.75\%), Bacteroidetes (42.08\%), Proteobacteria (12.25\%), Deferribacteres $(0.39 \%)$, Tenericutes $(0.20 \%)$, and others $(0.33 \%)$. In the model group, the composition was Firmicutes (20.54\%), Bacteroidetes (74.60\%), Proteobacteria (4.55\%), Deferribacteres $(0.03 \%)$, Tenericutes $(0.05 \%)$, and others $(0.23 \%)$. Bacteroidetes was the most abundant phylum $(47.60 \%)$ in the protection group with mixed LAB, which is similar to the levels observed in the control group. In the treatment group, especially mixed $\mathrm{LAB}$, the sum of the relative abundance of Firmicutes (62.58\%) and Bacteroidetes (89.89\%; 62.58 and $27.31 \%$, resp.), was similar to that in the control group $(86.83 \%)$. The results showed that, in the protection group and the treatment group, the flora structure in the mixed lactic acid bacteria group was the closest to the control group.

\section{Discussion}

S. aureus is a major human pathogen that causes intestinal infections, and several molecules produced by $S$. aureus cause a strong inflammatory response on the cell surface [4]. After the attack is recognized by TLR2, the corresponding cascade signal transduction is promoted, ultimately leading to the induction of cytokine production and other related immune responses [16]. Lactobacillus spp. are normal components of human intestinal flora [17] and can regulate the body's 


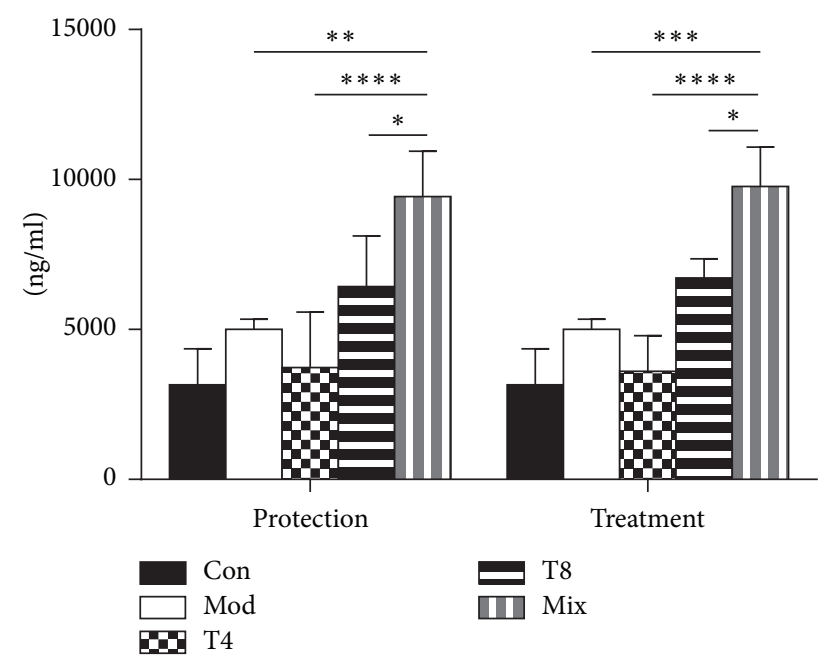

FIGURE 3: Intestinal sIgA levels. The amount of sIgA was determined by ELISA. Con: control group, Mod: mice infected with $S$. aureus without a week-long intervention with lactic acid bacteria. T4, T8: $L$. plantarum strains, Mix: mixed LAB. ${ }^{*} P<0.05,{ }^{* *} P<0.01,{ }^{* * *} P<$ 0.001 , and ${ }^{* * * *} P<0.0001$ versus mix.

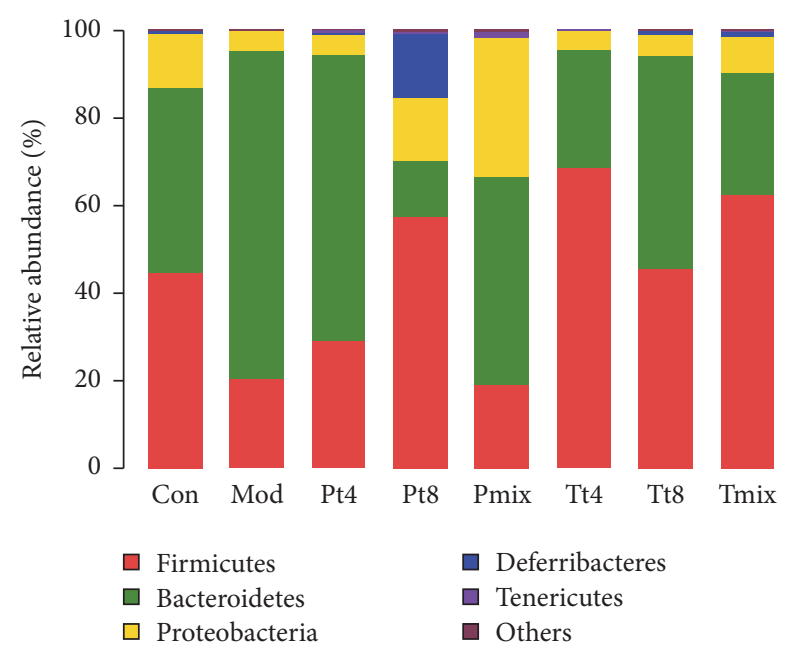

FIgURE 4: The microbiome composition in different study groups. Each bar represents the average relative abundance of bacterial taxa within a group. Con: control group; Mod: model group; P: protection group; T: treatment group; T4, T8: L. plantarum strains; Mix: mixed LAB.

immune system [10] to promote local immune prophylaxis and induction of anti-inflammatory factors [11]. In this study, L. plantarum (T4 and T8) were screened by in vitro antibacterial test and used to study the protective effects of LAB during S. aureus infection.

Earlier studies have shown that mice infected with pathogenic bacteria have a greater loss in body weight and that probiotic feeding-infected mice exhibit minimal loss of body weight [12]. In this study, the lactic acid bacteria mixed group of the protection arm and control group mice exhibited the same trends in weight gain; meanwhile, the mixed LAB group in the treatment arm had no significant changes in body weight. Wan et al. showed that LAB can inhibit infections of the liver, spleen, and other organs in mice [18]. The liver index of the protection group was similar to the control group; meanwhile, the mice infected with $S$. aureus exhibited hepatomegaly. The liver index was decreased by intervention with LAB but was still higher than in the control group. Our results show that LAB can prevent liver enlargement in mice infected with $S$. aureus.

Th1 mediates cellular immunity, which is able to capture intracellular bacteria, viruses, and cancer, inducing the secretion of IFN- $\gamma$ cytokines, a Th2 response to humoral immunity, and IL-4 secretion [19]. Studies have shown that serum IFN- $\gamma$ content is higher in mice following $S$. aureus infection [7]. Activate LAB increase Th1 type IFN- $\gamma$ cytokine secretion in spleen cells of mice infected with $S$. aureus while reducing the secretion of Th2 type IL- 4 cytokines [11, 20]. In our study, serum IFN- $\gamma$ levels were higher in the infection group compared with the control; meanwhile, the secretion of IL-4 decreased. Kemgang et al. showed that serum IL4 levels (i.e., Th2 cells) increased in the late stage of LAB intervention [12]. Other studies have shown that IL-4 is the key to the survival and proliferation of T cells, as IL4 promotes the production of Th2 cells and excessive IL4 inhibits the production of IFN- $\gamma$ [21]. In this study, the recovery of IFN- $\gamma$ decreased and IL- 4 increased. As a result, the ratio of IFN- $\gamma / \mathrm{IL}-4$ in the serum of mice treated with mixed $\mathrm{LAB}$ was reduced in the protection and treatment groups between infection and recovery. Our results show that mixed LAB inhibit the production of inflammation in $S$. aureus infected mice.

$\operatorname{sIg} \mathrm{A}$ is an immune barrier that prevents the adhesion and penetration of toxins, intestinal bacteria, and intestinal epithelial cells. Additionally, slgA inhibits allergenic and pathogenic microorganisms $[22,23]$ and prevents bacteria from destroying the intestinal mucosa. Jiang et al. showed that sIgA levels in the intestinal tract of infected mice are significantly higher than in controls [24]. In our study, sIgA levels in the intestinal contents of mice treated with LAB were significantly higher in the protection and treatment groups than in the control group, particularly in the mixed LAB arms. This may be due to the promotion of slgA secretion by LAB [12].

There are many microbial species in the intestines. Firmicutes are linked to the ability to harvest energy and absorb nutrients from food [25]. Interestingly, cluster analysis of operational taxonomic units showed administration of L. plantarum ZDY2013 for 3 weeks significantly increased the abundance of Proteobacteria [26]. In our study, the abundance of Proteobacteria in the intestine of mice treated with mixed LAB in the protection group also increased; meanwhile, the levels of Deferribacteres in the Lactobacillus plantarum T8 group were significantly higher than in the other groups. Deferribacteres have not been extensively studied, and their specific function is unknown. Xie et al. showed that oral administration of a probiotic resulted in an important, yet transient, alteration in the small intestinal microbiota that should confer a beneficial effect on the host [26]. Based on our results, mixed LAB have a potentially 
probiotic effect on the intestinal microbiome of mice infected with S. aureus.

\section{Conclusion}

Mixed LAB restored normal growth in mice following $S$. aureus infection, increased levels of the immune-related anti-inflammatory factor IL-4, and promoted secretion of sIgA. These results indicate that mixed LAB could resolve inflammation and promote a healthy microbiome in mice infected with $S$. aureus. This provides evidence for the use of probiotics for treatment of $S$. aureus infections.

\section{Conflicts of Interest}

All authors state that they have no conflicts of interest.

\section{Authors' Contributions}

Dayong Ren and Shengjie Gong contributed equally to this paper. Dayong Ren, Shengjie Gong, Jingyan Shu, and Ping Chen conceived and designed the experiments. Shengjie Gong, Jianwei Zhu, Fengjun Rong, Zhenye Zhang, and Di Wang performed the experiments. Liangfeng Gao, Tianming $\mathrm{Qu}$, and Hongyan Liu analyzed the data. Dayong Ren, Shengjie Gong, and Ping Chen wrote the manuscript.

\section{Acknowledgments}

This study was supported by the National Natural Science Foundation of China (31401475) and the Fund Project of Jilin Provincial Department of Education [2015 (no. 196)].

\section{References}

[1] B. M. Bröker, S. Holtfreter, and I. Bekeredjian-Ding, "Immune control of Staphylococcus aureus - Regulation and counterregulation of the adaptive immune response," International Journal of Medical Microbiology, vol. 304, no. 2, pp. 204-214, 2014.

[2] X. Liu, Z. Lei, D. Liu, and Z. Wang, "Development of a sandwiched microarray platform for studying the interactions of antibiotics with Staphylococcus aureus," Analytica Chimica Acta, vol. 917, pp. 93-100, 2016.

[3] M. Rezaei, R. Moniri, and S. G. A. Mousavi, "Molecular analysis and susceptibility pattern of meticillin-resistant Staphylococcus aureus strains in emergency department patients and related risk factors in Iran," Journal of Hospital Infection, vol. 89, no. 3, pp. 186-191, 2015.

[4] L.-Y. Hsu, L. Wijaya, and T.-H. Koh, "Community-associated meticillin-resistant Staphylococcus aureus," The Lancet, vol. 376, no. 9743, p. 767, 2010.

[5] M. Alimolaei, M. Golchin, and H. Daneshvar, "Oral immunization of mice against Clostridium perfringens epsilon toxin with a Lactobacillus casei vector vaccine expressing epsilon toxoid," Infection, Genetics and Evolution, vol. 40, pp. 282-287, 2016.

[6] C. Ghosh and B. Bishayi, "Toll-like receptor 2 and 6 interdependency in the erosive stage of Staphylococcus aureus induced septic arthritis mediated by IFN- $\gamma$ and IL-6 - A possible involvement of IL-17 in the progression of the disease," Immunobiology, vol. 220, no. 7, pp. 910-923, 2015.

[7] S. Mahanti, A. Majhi, K. Kundu, A. Basu, and B. Bishayi, "Systemic Staphylococcus aureus infection in restraint stressed mice modulates impaired immune response resulting in improved behavioral activities," Journal of Neuroimmunology, vol. 288, pp. 102-113, 2015.

[8] M. Godínez-Victoria, M. E. Drago-Serrano, H. Reyna-Garfias et al., "Effects on secretory IgA levels in small intestine of mice that underwent moderate exercise training followed by a bout of strenuous swimming exercise," Brain, Behavior, and Immunity, vol. 26, no. 8, pp. 1300-1309, 2012.

[9] K. B. Crossley, K. K. Jefferson, G. L. Archer, and V. G. Fowler, "Staphylococci in Human Disease: Second Edition," Staphylococci in Human Disease: Second Edition, pp. 1-623, 2009.

[10] J. H. Y. Jeong, S. Jang, B. J. U. Jung et al., "Differential immunestimulatory effects of LTAs from different lactic acid bacteria via MAPK signaling pathway in RAW 264.7 cells," Immunobiology, vol. 220, no. 4, pp. 460-466, 2015.

[11] W.-H. Lin, C.-R. Wu, H.-Z. Lee et al., "Induced apoptosis of Th2 lymphocytes and inhibition of airway hyperresponsiveness and inflammation by combined lactic acid bacteria treatment," International Immunopharmacology, vol. 15, no. 4, pp. 703-711, 2013.

[12] T. S. Kemgang, S. Kapila, V. P. Shanmugam, S. Reddi, and R. Kapila, "Fermented milk with probiotic Lactobacillus rhamnosus S1K3 (MTCC5957) protects mice from salmonella by enhancing immune and nonimmune protection mechanisms at intestinal mucosal level," Journal of Nutritional Biochemistry, vol. 30, pp. 62-73, 2016.

[13] N. Yuki, K. Watanabe, A. Mike et al., "Survival of a probiotic, Lactobacillus casei strain Shirota, in the gastrointestinal tract: selective isolation from faeces and identification using monoclonal antibodies," International Journal of Food Microbiology, vol. 48, no. 1, pp. 51-57, 1999.

[14] A. Rizzo, M. Fiorentino, E. Buommino, G. Donnarumma, A. Losacco, and N. Bevilacqua, "Lactobacillus crispatus mediates anti-inflammatory cytokine interleukin-10 induction in response to Chlamydia trachomatis infection in vitro," International Journal of Medical Microbiology, vol. 305, no. 8, pp. 815827, 2015.

[15] C. Bujalance, M. Jiménez-Valera, E. Moreno, M.-D. RuizLópez, A. Lasserrot, and A. Ruiz-Bravo, "Lack of correlation between invitro antibiosis and invivo protection against enteropathogenic bacteria by probiotic lactobacilli," Research in Microbiology, vol. 165, no. 1, pp. 14-20, 2014.

[16] N. W. J. Schröder, S. Morath, C. Alexander et al., "Lipoteichoic acid (LTA) of Streptococcus pneumoniae and Staphylococcus aureus activates immune cells via Toll-like receptor (TLR)-2, lipopolysaccharide-binding protein (LBP), and CD14, whereas TLR-4 and MD-2 are not involved," The Journal of Biological Chemistry, vol. 278, no. 18, pp. 15587-15594, 2003.

[17] J.-Y. Jung, J.-S. Shin, S.-G. Lee et al., "Lactobacillus sakei K040706 evokes immunostimulatory effects on macrophages through TLR 2-mediated activation," International Immunopharmacology, vol. 28, no. 1, pp. 88-96, 2015.

[18] M. L. Y. Wan, P. C. Turner, K. J. Allen, and H. El-Nezami, "Lactobacillus rhamnosus GG modulates intestinal mucosal barrier and inflammation in mice following combined dietary exposure to deoxynivalenol and zearalenone," Journal of Functional Foods, vol. 22, pp. 34-43, 2016. 
[19] M. H. Boskabady, S. Shahmohammadi Mehrjardi, A. Rezaee, H. Rafatpanah, and S. Jalali, "The impact of Zataria multiflora Boiss extract on in vitro and in vivo Th1/Th2 cytokine (IFN- $\gamma /$ IL4) balance," Journal of Ethnopharmacology, vol. 150, no. 3, pp. 10241031, 2013.

[20] D. Ghadimi, M. de Vrese, K. J. Heller, and J. Schrezenmeir, "Lactic acid bacteria enhance autophagic ability of mononuclear phagocytes by increasing Thl autophagy-promoting cytokine (IFN- $\gamma$ ) and nitric oxide (NO) levels and reducing Th2 autophagy-restraining cytokines (IL-4 and IL-13) in response to Mycobacterium tuberculosis antigen," International Immunopharmacology, vol. 10, no. 6, pp. 694-706, 2010.

[21] H. Kang, J. S. Moon, M.-G. Lee, and N. S. Han, "Immunomodulatory effects of Leuconostoc citreum EFEL2061 isolated from kimchi, a traditional Korean food, on the Th2 type-dominant immune response in vitro and in vivo," Journal of Functional Foods, vol. 20, pp. 79-87, 2016.

[22] Z. Wu, D. Pan, Y. Guo, and X. Zeng, "N-acetylmuramic acid triggers anti-inflammatory capacity in LPS-induced RAW 264.7 cells and mice," Journal of Functional Foods, vol. 13, pp. 108-116, 2015.

[23] J. Baumann, C. G. Park, and N. J. Mantis, "Recognition of secretory IgA by DC-SIGN: implications for immune surveillance in the intestine," Immunology Letters, vol. 131, no. 1, pp. 59-66, 2010.

[24] Q. Jiang, H. Zhang, Y. Xie, and Y. Wang, "Recombinant expression of porcine lactoferrin peptide LF-6 with intein technology and its immunomodulatory function in ETEC K88-infected mice," International Immunopharmacology, vol. 39, pp. 181-191, 2016.

[25] W. Wang, J. Cao, J.-R. Li, F. Yang, Z. Li, and L.-X. Li, "Comparative analysis of the gastrointestinal microbial communities of bar-headed goose (Anser indicus) in different breeding patterns by high-throughput sequencing," Microbiological Research, vol. 182, pp. 59-67, 2016.

[26] Q. Xie, M. Pan, R. Huang et al., "Short communication: modulation of the small intestinal microbial community composition over short-term or long-term administration with Lactobacillus plantarum ZDY2013," Journal of Dairy Science, vol. 99, no. 9, pp. 6913-6921, 2016. 

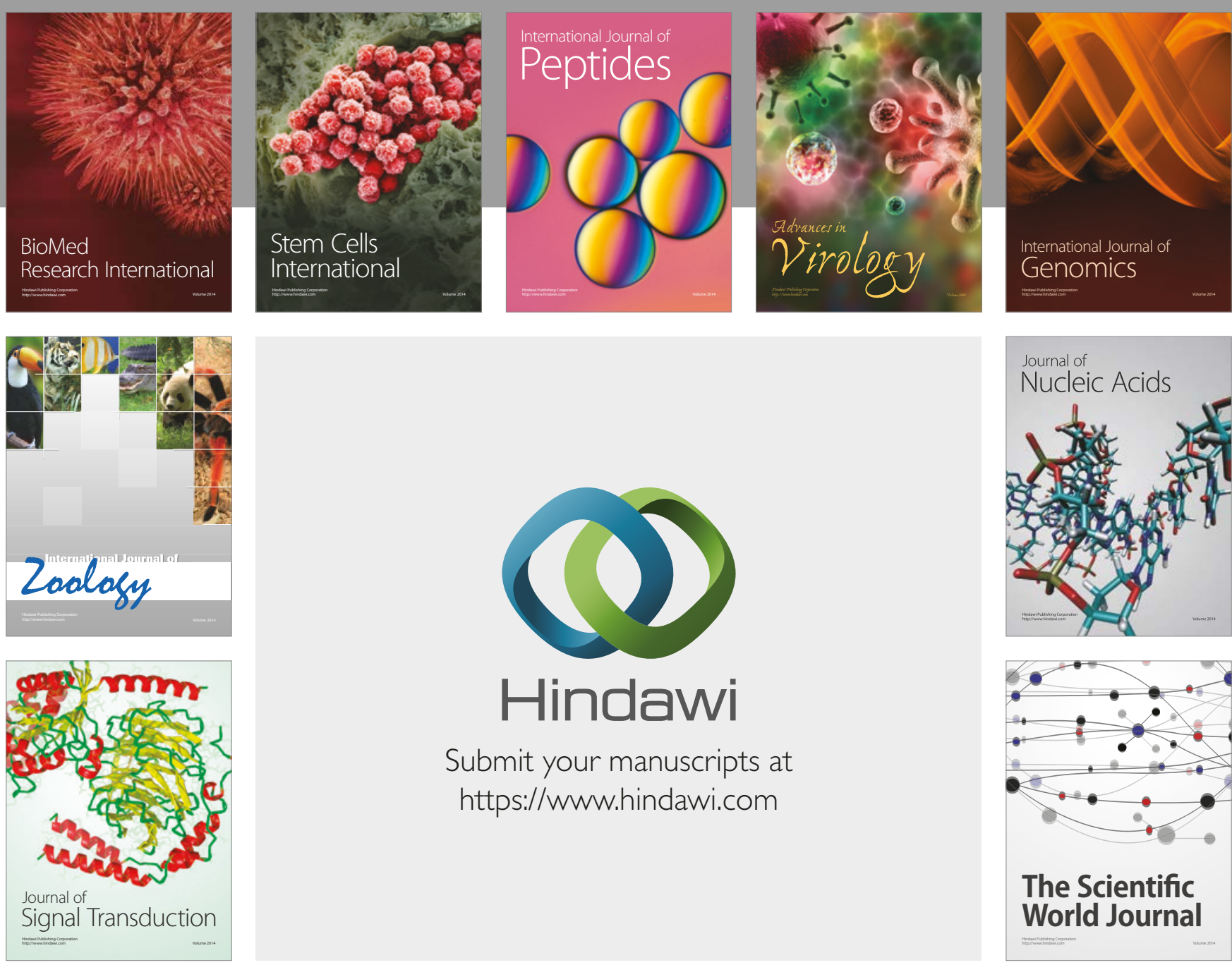

Submit your manuscripts at

https://www.hindawi.com
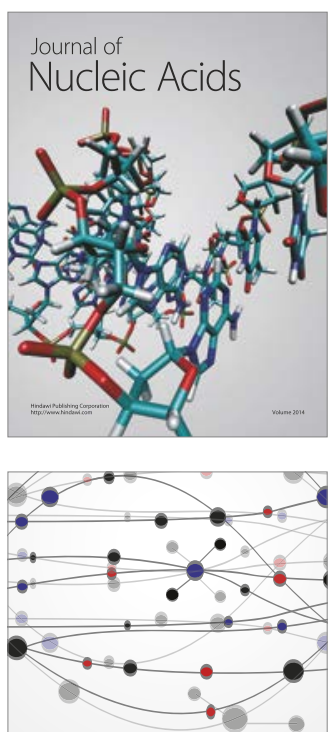

The Scientific World Journal

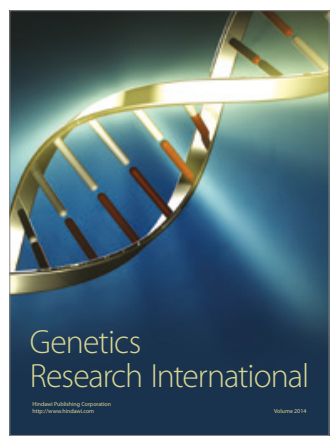

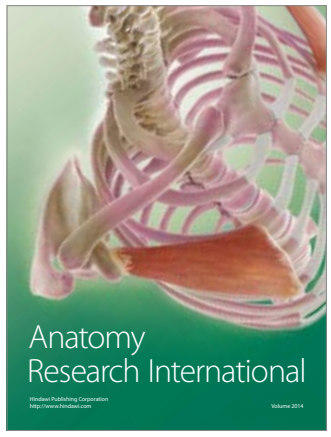

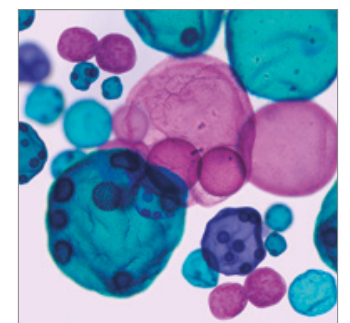

International Journal of Microbiology
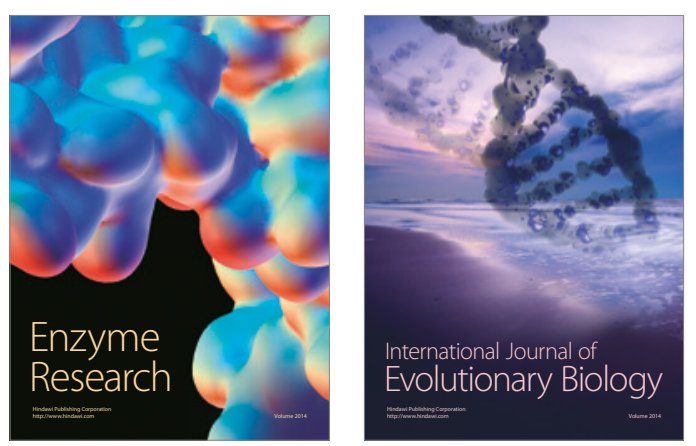
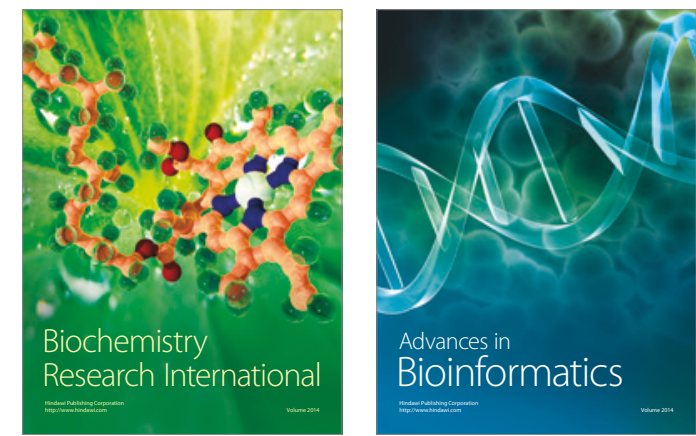

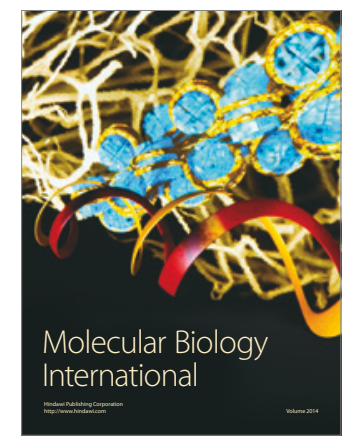

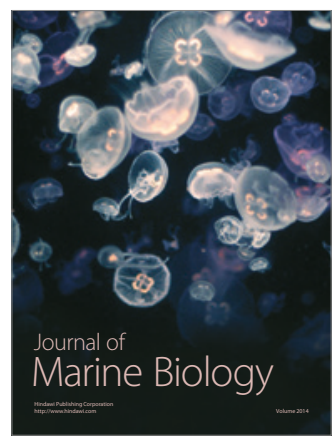

